\title{
Results of Crust and Mantle Soundings in Central and Northern Europe in the 21st Century (Review)
}

\author{
Vladimir Yu. SEMENOV \\ Institute of Geophysics, Polish Academy of Sciences, Warszawa, Poland \\ e-mail: sem@igf.edu.pl
}

\begin{abstract}
The first decade of 21 st century is characterized by the appearance of new approaches to deep induction soundings. The theory of magnetovariation and magnetotelluric soundings was generalised or corrected. Spatial derivatives of response functions (induction arrows) were obtained for the ultra-long periods. New phenomena have been detected by this method: secular variations of the Earth's apparent resistivity and the rapid changes of induction arrows over the last 50 years. The first one can be correlated with the number of earthquakes, and the second one with geomagnetic jerks in Central Europe. The extensive studies of geoelectrical structure of the crust and mantle were realized in the frame of a series of international projects. New information about geoelectrical structures of the crust in Northern Europe and Ukraine was obtained by deep electromagnetic soundings involving controlled powerful sources. An influence of the crust magnetic permeability on the deep sounding results was confirmed.
\end{abstract}

Key words: induction soundings, conductivity, crust, mantle, Europe.

\section{INTRODUCTION}

The end of the 20th century was characterized by an increasing interest in the deep induction soundings of the Earth mantle, particularly by the magnetovariation (MV) method (e.g., Roberts 1984, 1986, Schultz and Larsen 1987, 1990, Semenov 1989, Schultz 1990, Schultz et al. 1993, Olsen 1992,

Ownership: Institute of Geophysics, Polish Academy of Sciences;

(C) 2015 Semenov. This is an open access article distributed under the Creative Commons Attribution-NonCommercial-NoDerivs license,

http://creativecommons.org/licenses/by-nc-nd/3.0/. 
1998, 1999a, Semenov and Jóźwiak 1999, Schmucker 1999a,b) and using satellite data (e.g., Oraevsky et al. 1993, Olsen 1999b). A feature of deep soundings is the lack of direct verification of the obtained results except of the ultra-deep borehole data in the Earth's crust, reaching $12 \mathrm{~km}$ depth. Therefore, the correctness of the induction sounding theory plays a dominant role in such investigations. For example, it is obvious that the model of the source field in the common form of a "plane wave" will not be valid for long periods as well as without sphericity of the Earth taken into account (Schultz and Zhang 1994).

In retrospect, it is surprising that some oldest works in geoelectricity were broader than the traditional "plane wave" model. The concept of impedance was introduced in the early 1930s by Leontovich in Russia. Then his student Rytov (1940) published the mathematical model in France and Russia, the first approximations of which are applied now as magnetotelluric (MT) and generalized magnetovariation (MV) soundings. Later a similar work was published by Wait (1954). Leontovich (1948) has considered limits of applicability of the Rytov model and finally Senior and Volakis (1995) found a small error in that work. The simplified model suggested by Tikhonov (1950) and Cagniard (1953) certainly had a great success for the exploration of mineral resources by the MT method. Two traditional MV methods for estimation impedances and tippers separately required a separation of the observed field to "normal" and "anomalous" parts; that is a vague procedure. Solution of the problem has been already incorporated in the Rytov model that was pointed out by Guglielmi and Gokhberg (1987), while the simplified MV method was already developed and used by Banks (1969), Berdichevsky et al. (1969), and Schmucker (1970). Approaches considered by Bates et al. (1976), Woods and Lilley (1979), Kuckes (1973), and Kuckes et al. (1985) were closer to Rytov's one. The corrected concept for the induction sounding was proposed by Shuman (1999). The theoretical transformations of impedance matrix (called "tensor" with overstatement because we do not know exactly what kinds of field sources formed the measured signal in each direction) to an "resistivity azimuthal tensor" was obtained theoretically by Reilly (see Weckmann et al. 2003) and Semenov (1988). As a result, we have polar diagrams of the apparent resistivity instead of impedances. They can be not equal, as shown below.

The deep induction soundings request impedances in a wide period range to obtain reliable results. To satisfy this request, the joint inversions of local MT impedances with regional MV responses were tested by Semenov (1988), Egbert and Booker (1992), Schultz et al. (1993) and Semenov and Rodkin (1996). This approach was used for the induction soundings in the frame of the following international projects: Baltic Electromagnetic Array Research (BEAR, 1998-2002), Central Europe Mantle Electrical Structure 
(CEMES, 2001-2003), Electro-Magnetic soundings of Trans-European Suture Zone" (EMTESZ, 2003-2005), Electro-Magnetic Mini Arrays (EMMA, 2005-2008) in Fennoscandia, Fennoscandian Electrical conductivity from soundings with Natural and Controlled Sources (FENICS, 2007-2009), and Lithospheric Structure of TESZ by Magneto-Variation Soundings (LS-MVS, 2009-2012) including territories of Belarus, Czech Republic, Finland, Germany, Hungary, Norway, Poland, Romania, Russia, Slovakia, Sweden, and Ukraine. The national and global investigations have been already reviewed by Korja (2007) and Kuvshinov (2012).

\section{THEORY AND MODELING}

In the beginning of 21 st century, a number of important theoretical works were published devoted to basic problems of the deep MV and MT soundings. The first problem concerned the deep MV sounding. The common model with the plane wave did not contain a vertical component of the magnetic field, which only occurred in the presence of heterogeneity in the medium. However, the long-period field of an auroral electrojet can be considered as a plane wave in the middle latitudes (Vanyan et al. 2002) but naturally with the vertical component. The deep soundings are also possible using the source, but there is a problem of separating the observed field into parts related and not related to the presence of inhomogeneities without knowledge about medium properties. Solution to this problem follows from several old and recent works.

Following Rytov (1940) and Wait (1954), the scalar response function $C(\mathbf{r}, \omega)$ and its gradient can be found simultaneously from the boundary condition derived by Guglielmi and Gokhberg (1987):

$$
i \omega B_{z}=Z(\omega, \mathbf{r}) \cdot \operatorname{div} B_{\tau}+\operatorname{grad} Z(\omega, \mathbf{r}) \cdot \boldsymbol{B}_{\tau},
$$

where $B_{z}$ and $\boldsymbol{B}_{\tau}$ are the Fourier amplitudes of the "observed" vertical and tangential component of magnetic field, respectively; $Z(\omega, \mathbf{r})=E_{x} / B_{y}$ is the scalar impedance, which can be recalculated in a response function $C(\omega, \mathbf{r})=\mathrm{Z} / i \omega$. Here $E_{x}$ is the electric field component orthogonal to $B_{y}, \omega$ is the angular frequency, $i$ is the imaginary unit, and $\mathbf{r}$ is the position vector. Relation 1 without the term $\operatorname{grad} Z \cdot \boldsymbol{B}_{\tau}$ is known as the horizontal spatial gradient (HSG) method and used for soundings of the laterally homogeneous media (Logvinov 2002). Its variant for the linearly polarized field generated by the magnetospheric ring currents was named the geomagnetic depth sounding (GDS) method (Olsen 1992) and used in the spherical geomagnetic reference system $(r, \varphi, \theta)$ for soundings of the mid- and lower mantle: $B_{\mathbf{r}}=$ $C(\omega, \mathbf{r}) \cdot 2 \cdot B_{\theta} / R \cdot \operatorname{tg} \theta$. Here $R$ is the Earth's radius; $\theta$ is the co-latitude. Note that the response function $C(\omega, \mathbf{r})$ in the GDS method has a direction 
coinciding with an averaged direction of the induced currents, i.e., along the geomagnetic longitudes and thus could be considered as a vectoral value. A new interesting transfer function $D(\omega) \sim B_{\varphi}(\omega) / B_{\theta}(\omega)$ for the GDS method has been proposed by Fujii and Schultz (2002) along with the conventional response $C(\omega) \sim B_{r}(\omega) / B_{\theta}(\omega)$. The physical sense of the response $D(\omega)$ is fixing the observed deviations of horizontal field from the geomagnetic coordinate system. The function $D(\omega) \neq 0$ can be caused by some inhomogeneity in the conductive Earth or changing of conductivity due to changes of a relative position of ring currents and the Earth, during the magnetic storms for example.

Following Schmucker (2003), the magnetic field $\mathbf{B}(t)$ may be separated into "normal" (n) and "anomalous" (a) parts. The "normal" part can be applied for soundings of a laterally homogeneous medium while the "anomalous" part is used to fix anomalous zones in media characterized by the complex tippers $a(\omega, \mathbf{r})$ and $b(\omega, \mathbf{r})$ in the empirical relation $B_{z \mathrm{a}}=a \cdot B_{x}+$ $b \cdot B_{y}$. Because the "observed field" $B_{z}=B_{z \mathrm{n}}+B_{z \mathrm{a}}$, this and HSG equations were combined by Schmucker into the single one:

$$
B_{z}=C(\omega, \mathbf{r}) \cdot \operatorname{div} \boldsymbol{B}_{\mathrm{n} \tau}+c \cdot B_{x}+d \cdot B_{y} .
$$

The response function was considered here as a tensor. Equation 2 is similar to Eq. 1 if $\operatorname{div} \boldsymbol{B}_{\tau} \equiv \operatorname{div} \boldsymbol{B}_{n \tau}$. Besides, the tippers $a(\omega, \mathbf{r}), b(\omega, \mathbf{r})$, and the functions $c(\omega, \mathbf{r})=\partial C / \partial x, d(\omega, \mathbf{r})=\partial C / \partial y$ are essentially different and accordingly the conventional induction arrows $\boldsymbol{C}_{u}, \boldsymbol{C}_{v}$ differ from the vectors $\boldsymbol{S}_{u}$ and $\boldsymbol{S}_{v}$, based on $c$ and $d$ values. Relation 2 has been applied to the mantle soundings by Schmucker (2003).

The problem has been considered by Shuman and Kulik (2002), and Shuman (2007). New precise boundary conditions were suggested:

$$
B_{z}=C \operatorname{div} \boldsymbol{B}_{\tau}+\operatorname{grad} C \cdot \boldsymbol{B}_{\tau}+C_{0}^{*} \operatorname{div}\left(\boldsymbol{B}_{\tau}^{*} \times \mathbf{n}\right)+\operatorname{grad} C_{0}^{*}\left(\boldsymbol{B}_{\tau}^{*} \times \mathbf{n}\right) .
$$

Here the asterisk $\left(^{*}\right)$ means the complex conjugate value; $C$ and $C_{0}$ are two different functions, $\mathbf{n}$ is the unit vector normal to the surface. The question may arise: why Eq. 3 is so long? Equations 1-3 are the impedance boundary conditions on the Earth's surface (Senior and Volakis 1995). The boundary condition 3 is based on a more rigorous mathematical approach determining new impedances from the "vectoral" impedance boundary condition (VIBC) introduced by Aboul-Atta and Boerner (1975). They used the theorem that the electromagnetic fields inside a medium can be found uniquely if the tangential fields are known on its "full closed surface" (Harrington 1961). The VIBC are generalizing the complex Fourier spectra $\boldsymbol{E}(\omega)$ and $\boldsymbol{B}(\omega)-$ actually included in the boundary conditions. In other words, two projections of the complex vector $\boldsymbol{E}_{\tau}(\omega)$ on the complex vector $\boldsymbol{B}_{\tau}(\omega)$ require two orthogo- 
nal bases in a 2D unitary vector space and consequently two impedances must be considered simultaneously (Shuman 2003).

Omitting the part with response $C_{0}$ in the Eq. 3 and rewriting the expression 2 in the corresponding form with gradient of $C(\omega, \mathbf{r})$ there can be noticed a similarity of all three approaches $(1,2$, and 3$)$. The neglect of $C_{0}$ is caused by the necessity of determining six unknown transfer functions from the single equation in practice. It is seen that the physical sense of tippers and induction arrows is the spatial derivatives of the response function along the horizontal axis. By the way, the approaches 2 and 3 were presented by Prof. Schmucker and Prof. Shuman separately on the same day at the international conference in Poland (in 2001). Audience, including the author of this work, did not recognize at first the similarity of these reports.

Impedances depend in general on the particular sounding methods, on the properties of the conductive medium including their magnetic permeability, as well as on the adopted model of the space in the theory, the latter aspect being important while considering the way in which the induced currents are locking, even at infinity.

Moreover, the impedances depend on the exciting field that originates from sources of several different types in the period range of hours to 11 years used for the mantle electromagnetic soundings. Thus, quoting Guglielmi and Gokhberg (1987) we can say that "the Earth does not have its own impedance".

(a)

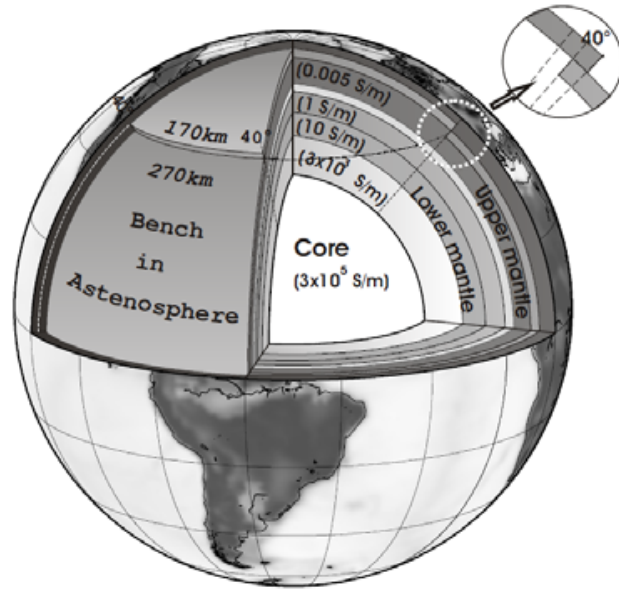

(b)

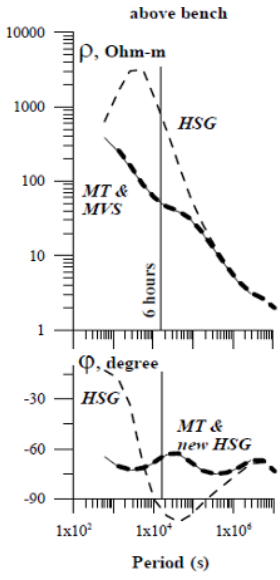

(c)

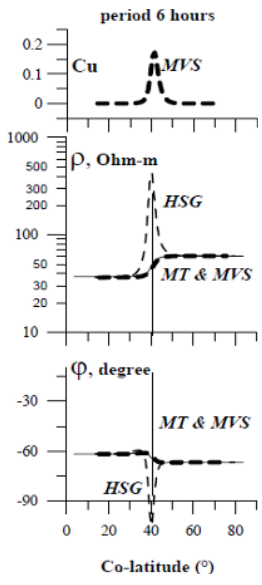

Fig. 1: (a) The model of the Earth's interiors with the surface shell conductance; (b) the apparent resistivity and impedance phase spectra obtained by MT - the thin line, HSG (GDS) - the thin dash line, and MVS (GMV) - the thick dash line soundings just above the uplift of the mantle conductive layer; (c) the profile results along co-latitudes with real induction arrow (Cu) (after Vozar and Semenov 2010). 
The question arises: how great is the difference between the results obtained by different induction methods over heterogeneous media? The spherical modeling (Kuvshinov et al. 2005) was applied (Vozar and Semenov 2010) along the profile crossing the essential bench at co-latitude $40^{\circ}$ whose top changed from 170 to $270 \mathrm{~km}$ (Fig. 1a). The modeling of different types of responses above 2D and 3D mantle inhomogeneities has shown that the various methods can give mutually inconsistent results in the apparent resistivities. The results can reach an order for apparent resistivity modules exactly above the center of anomaly while phase difference can reach tenths of degrees (Fig. 1c). Besides, induction vectors are depending on spherical latitudes. However, the MT and GMV sounding methods have shown the identical apparent resistivities above the inhomogeneity!

\section{METHODS AND SOURCES}

Let us note that the magnetotelluric impedance $Z(\omega)$ for a fixed $\omega$ is a ratio $\boldsymbol{E}(\omega) / \boldsymbol{B}(\omega)$ because the measured magnetic field is the magnetic induction $\mathbf{B}(t)$, but not the field intensity $\mathbf{H}(t)$, as evidenced by its unit - nanotesla (equal to $1 \gamma$-off-system unit). Exactly this unit had been required by the IAGA in the resolution no. 3 of "Transactions..." (1973). Note that the impedance at a fixed period is a "functional" of the conductivity (Berdichevsky and Zhdanov 1981). It means that many distributions of conductivity can correspond to the impedance value found for a fixed $\omega$. This fact does not contradict the theorem of uniqueness of the inverse solution for induction soundings proved for "infinite input data" (Rokitjansky 1982).

Estimations of impedances in practice are based on the random process theory assuming impedances as transfer functions between spectra of observed field components. These transfer functions are usually considered as

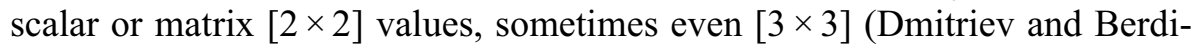
chevsky 2002). The last generalized heuristic approach leads to a similar result as obtained by Becken and Pedersen (2003). Such an approach is mixing both modes for the corresponding impedances that complicate analysis of data over inhomogeneous media (Semenov and Shuman 2010). Separating of the modes for 3D case has been discussed by Becken et al. (2008). Note that the impedances can be found in the time domain too (Nowożyński 2004).

The publication by Weckmann et al. (2003) brings us back to the problem of transforming the MT impedance matrix to apparent resistivity tensor elements $\rho_{i j}^{*}$ for a laterally anisotropic medium. Widespread approach is to consider the impedance as a tensor, two and sometimes four elements of which are recalculated into the same quantity of the scalar apparent resistivities even keeping indexes of the impedance tensor. Result of such a procedure can look very strange. It is more natural to consider "resistivity" 
of media as an "azimuthal tensor" and impedances as a matrix (for MT), vector (for GDS) or scalar (for GMV). The theoretical evidence transforming an impedance matrix to the resistivity tensor made by both Reilly (see Weckmann et al. 2003) and Semenov (2000) are identical:

$$
\begin{aligned}
& \rho_{x x}^{*}(\omega)=\left(Z_{x y}^{2}-Z_{x x} Z_{y y}\right) \mu / \omega ; \quad \rho_{x y}^{*}(\omega)=Z_{x x}\left(Z_{y x}-Z_{x y}\right) \mu / \omega ; \\
& \rho_{y y}^{*}(\omega)=\left(Z_{y x}^{2}-Z_{x x} Z_{y y}\right) \mu / \omega ; \quad \rho_{y x}^{*}(\omega)=Z_{y y}\left(Z_{x y}-Z_{y x}\right) \mu / \omega .
\end{aligned}
$$

Of course, reconsidering long-standing postulates is a thankless job. But frequent recording of $\rho_{x y}^{*} \sim Z_{x y}^{2}$ is without sense: asterisk $x y$ marks a minor element of resistivity tensor while it marked major element of impedance. The relation following from the theory is $\rho_{x x}^{*} \sim Z_{x y}^{2}-Z_{x x} Z_{y y}$. To obtain a scalar resistivity $\rho_{x x}^{*} \sim Z_{x y}^{2}$ or $\rho_{y y}^{*} \sim Z_{y x}^{2}$ the value of $Z_{x x} Z_{y y}$ must be minimized. Such directions were named the preferential ones; they may be not orthogonal (Fig. 2). The apparent resistivity modules shown here are only shifted at both preferential directions and their phases are similar (Fig. 2b), while they are different for the orthogonal principal directions. Moreover, the spectra of apparent resistivities obtained from the impedance matrix by common and theoretical transformation 4 can be essentially different from their 1D inversion models. A careful study of the anisotropic media with the arbitrarily directed tensor of conductivity has been made by Pek (2002) and Pek and Santos (2002).

The problem of deep soundings arises: how an obtained local MT resistivity tensor can be combined with the regional GMV or $S q$ scalar resistivity or with the GDS continental vectoral one? In order to combine

(a)

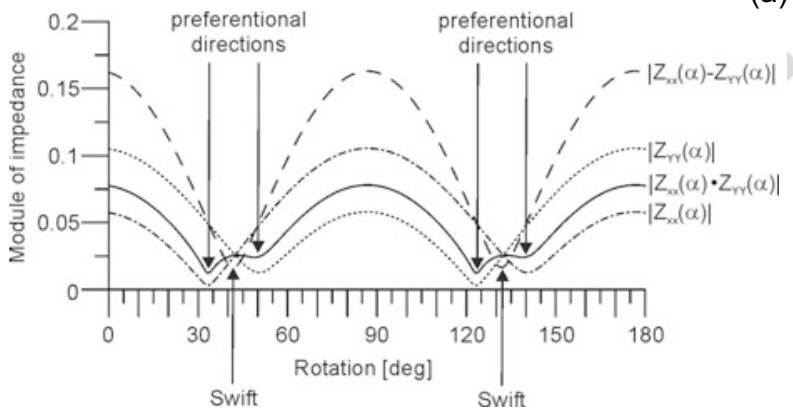

(b)

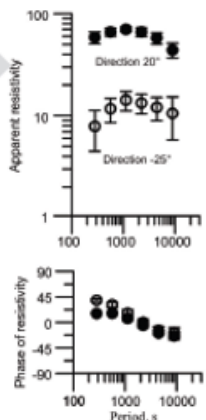

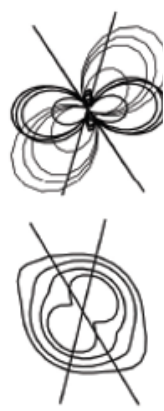

Fig. 2: (a) Choice of two preferential directions $\left(\min \left|Z_{x x} Z_{y y}\right|\right.$ ) in comparison with the orthogonal principal directions chosen by the Swift's procedure $\left(\min \left|Z_{x x}-Z_{y y}\right|\right)$; (b) example of apparent resistivities for the observatory HRB (after Semenov et al. 2008). 
them, we have to assume that a medium is rather homogeneous at great depths. But in fact the currents of different sources are induced and locked in different ways inside the Earth and may contain different information about its structure. This difference is clearly visible for the spherical model in Fig. 1 due to the different sources and methods including the $S q$ variations (Semenov et al. 2013). This problem still requires further efforts to study the deep irregularities in the mantle.

The problem of a lack of coincidence between inversion models of different teams in the international projects obtained from the same initial apparent resistivities is arising too. If it concerns $1 \mathrm{D}$ modeling, their results can be transformed to the monotonically increasing conductance with depth. This approach gives possibility to investigate large areas presenting results as schemes of conductance at a fixed depth or depths to a fixed conductance even without subsurface parts for comparison (Semenov and Jóźwiak 2006).

Besides, it was established that secular variations of the Earth apparent resistivity estimated by two GMV and GDS methods can reach $20 \%$ of the mean measured values (Fig. 3). These variations are well correlated with the number of earthquakes in the seismically active areas of Central Europe. The depths of the earthquakes are less than $40 \mathrm{~km}$ where sources of such variations are situated. So the internal as well as changing external sources can essentially disturb the sounding results during long observations. Besides, the induction arrows can change their directions and their values during a couple of years. This phenomenon coincides with the appearance of geomagnetic jerks in Central Europe (Petrishchev and Semenov 2013).

Another registered phenomenon is connected with the high magnetic permeability near the Curie point in the crust (Kiss et al. 2005). The influence of this effect on the deep soundings was considered by Szarka et al. (2007).
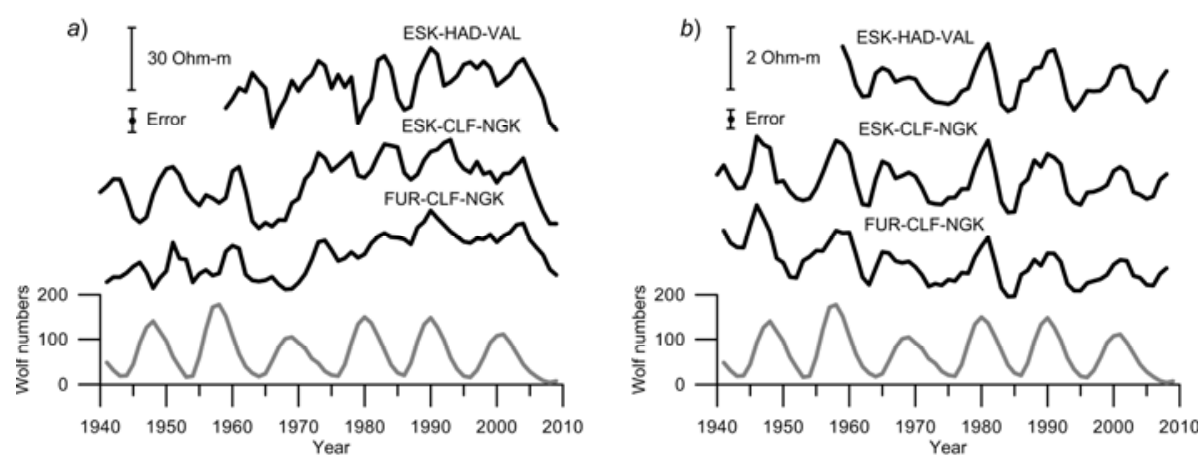

Fig. 3. Comparisons of the Earth's apparent resistivities with $T=8.8$ hours (a), and 30 days (b) observed by different groups of observatories marked by their codes (after Petrishchev and Semenov 2013). 


\section{RESULTS OF DEEP SOUNDINGS}

The final results of electrical conductivity studies of the Earth's crust at the Ukrainian and Fennoscandian shields were published by Ingerov et al. (1999) and Korja et al. (2002). Both studies show considerable heterogeneity in the crust conductance in these regions, reaching few orders of magnitude. The example of the crust conductance in the Ukraine is presented in Fig. 4.

The extensive deep geoelectric studies in Northern Europe were realized in the frame of two international projects: BEAR (1998-2002) with its deep sounding continuation - EMMA (2005-2008) in Fennoscandia. These measurements have been done using c.a. $50 \mathrm{MT}$ stations, displayed permanently over the $150 \times 150 \mathrm{~km}$ network on the territory of Fennoscandian shield. Four countries have taken part in the investigations. The obtained crustal conductance up to $60 \mathrm{~km}$ depth for Northern Europe is shown in Fig. 4b. The fixed conductance contrast reaches six orders and shows extremely high electrical heterogeneity of the crust in the Fennoscandian shield (Korja et al. 2002). The large regions of high resistance are surrounded by relatively narrow, highly conducting zones with conductances reaching dozens of $\mathrm{kS}$. The origin of crustal anomalies can be connected with electronically conducting sulfide and carbon bearing structures (Zhamaletdinov 1996). The upper mantle conductance was estimated from the BEAR data during the EMMA project. It was shown that at depths of $150-300 \mathrm{~km}$ the conductance reaches 4-5 kS that may be expressed as "an asthenosphere conducting layer" (Sokolova and Varentsov 2007).

The second project was CEMES (2001-2003) at the territories of seven countries. The long-time MT measurements have been carried out at eleven

(a)

(b)
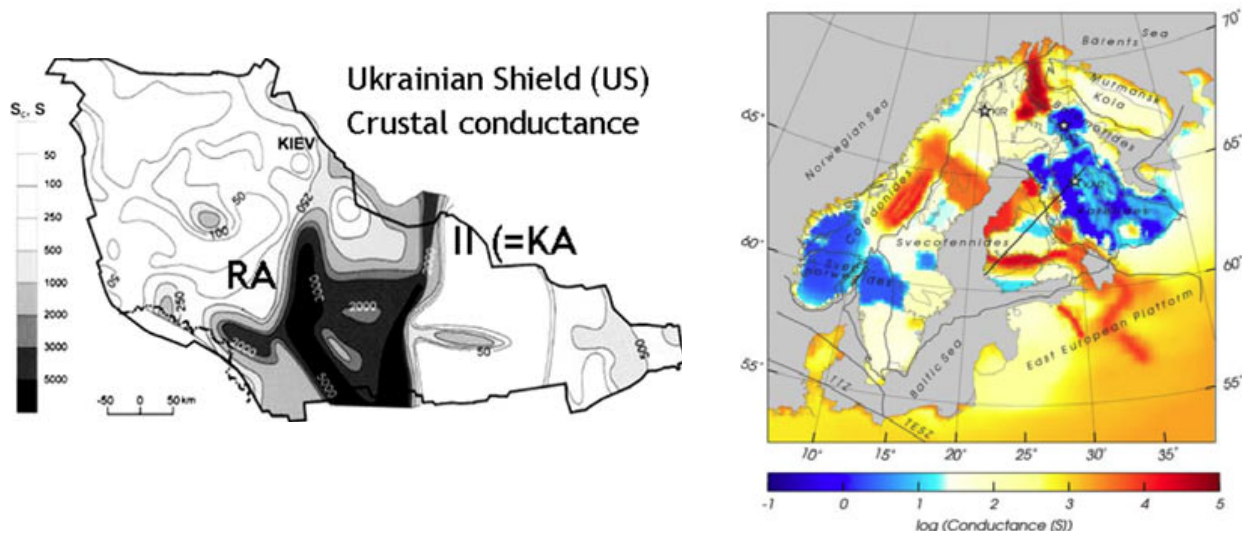

Fig. 4. Schemes of the crust conductances on the Ukrainian (a) and Fennoscandian (b) shields (after Ingerov et al. 2005 and Korja et al. 2002, respectively. 
(a)

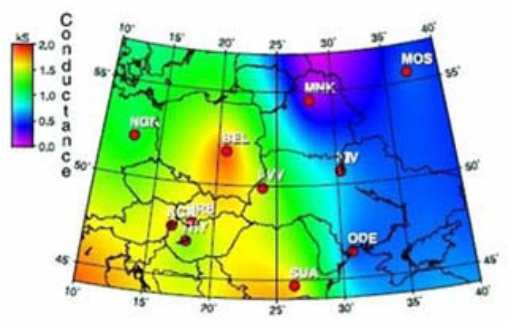

(c)

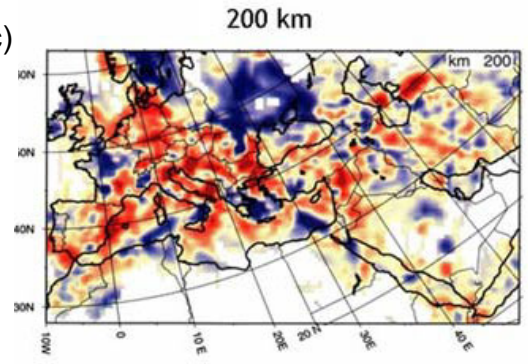

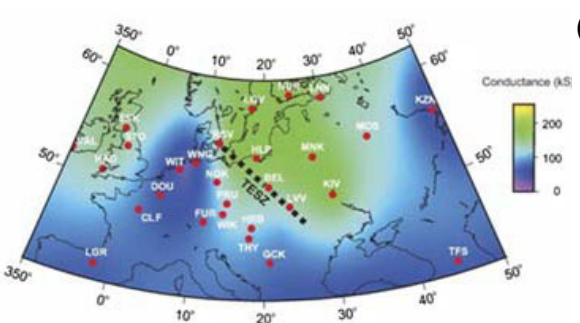

(b)

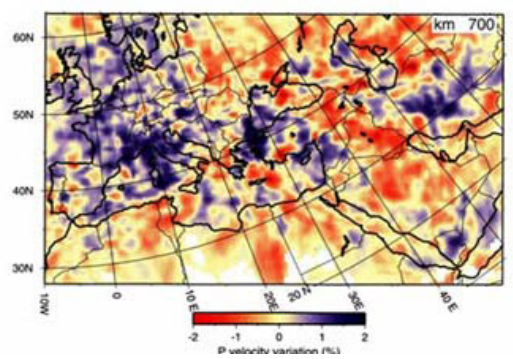

(d)

Fig. 5. Comparison of the conductance distributions in kS: depths of 50-200 km (a) and depths of 50-770 km (b) with seismic $P$ velocity variations (\%) at $200 \mathrm{~km}(\mathrm{c})$ and $700 \mathrm{~km}$ (d) (after Korja 2007).

geomagnetic observatories of Central Europe and their sounding results were combined with the MV soundings obtained at the same observatories using the historical hourly data (Fig. 5a).

Besides, the MV impedances for the periods 4 hours -11 years estimated by six authors for 35 European observatories were collected. The precise selection of their results and subsequent combination allowed applying 1D inversion modeling to estimate the regional mantle conductance at a depth of $770 \mathrm{~km}$ beneath chosen observatories. The scheme of interpolation is shown in Fig. 5b.

These results have shown that the Trans-European Suture Zone (TESZ) coincides with the depth gradients of the $1 \mathrm{kS}$ conductance in the upper mantle. The same effect has been observed along TESZ for the conductance gradient at a depth of $770 \mathrm{~km}$ in the mid-mantle. However, the conductance is increasing to the west in the upper mantle while in the mid-mantle it is increasing to the east. This reversing occurs by seismic data at about 600 $800 \mathrm{~km}$ and has been confirmed by deep sounding results obtained along profile Germany-Belarus (Fig. 6).

The international project EMTESZ (2003-2005) was carried out on the Polish and German territories. Two long magnetotelluric profiles along the seismic ones LT-7 (Guterch et al. 1994) and P2 (Janik et al. 2002) were 


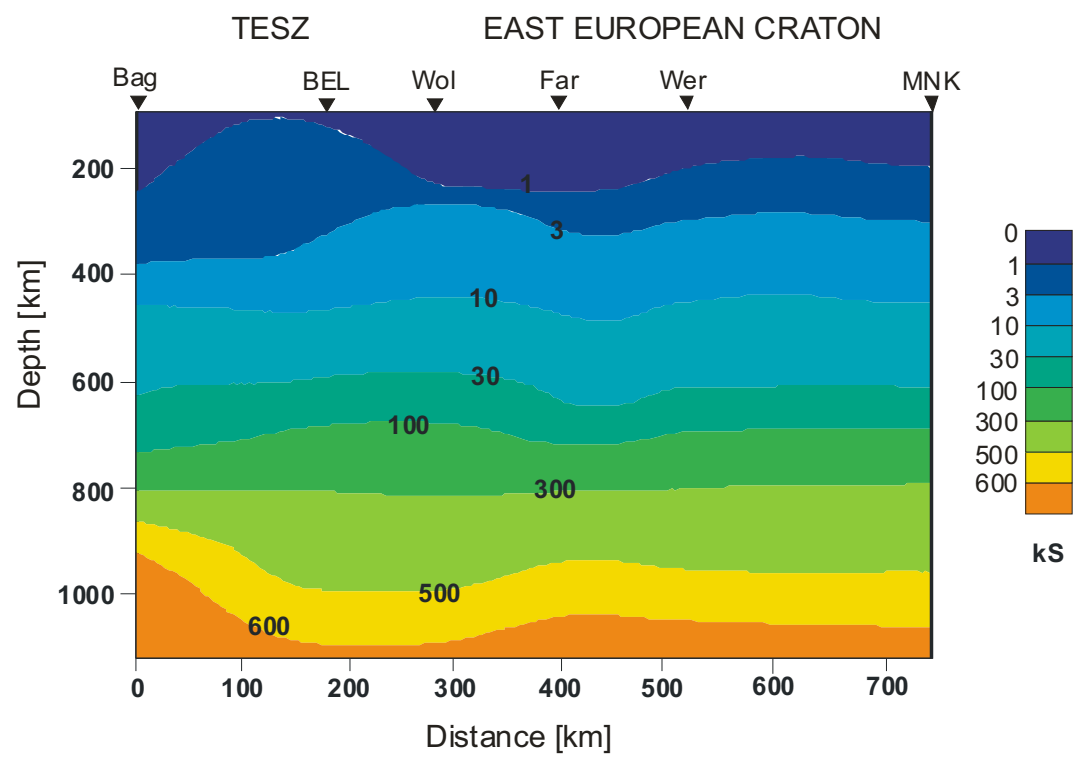

Fig. 6. Smoothed conductance deep structure along profile between geomagnetic observatories NGK (Germany) and MNK (Belarus) (after Semenov and Jóźwiak 2005).

studied by the MT method in the wide period range $\left(0.1-10^{4} \mathrm{~s}\right)$. The resistivity cross-sections up to upper mantle depths were published by Ernst et al. (2008).

Depths of the upper mantle were investigated separately combining three MT soundings in the TESZ-center of the profiles with apparent resistivities obtained at the nearest geomagnetic observatory Belsk situated in the TESZ too. The observed sounding result is a rarity: two curves of apparent resistivity in orthogonal directions are reaching each other at the period of one day (Fig. 7b). The obtained effect can be explained by the laterally anisotropic layer at depths of $30-40 \mathrm{~km}$.

The next project was the FENICS (2007-2009). Two mutually orthogonal industrial power transmition lines of 110 and $120 \mathrm{~km}$ lengths (Fig. 8) with the generator of $200 \mathrm{~kW}$ were used to estimate of the transversal resistance $T\left[\Omega \cdot \mathrm{m}^{2}\right]$ of the lithosphere in the frequency range $0.1-200 \mathrm{~Hz}$ (Zhamaletdinov et al. 2011). Spacing between transmitters and receiving points reached $700 \mathrm{~km}$. Besides, the soundings with the Magneto-HydroDynamic (MHD) generator "Khibiny" of $80 \mathrm{MW}$ power were carried out for the investigations (Zhamaletdinov 2005). The Moho depth gradient of the deepest anomalous zone in Europe (Grad et al. 2007) corresponds to the gradient of the transversal resistance. 


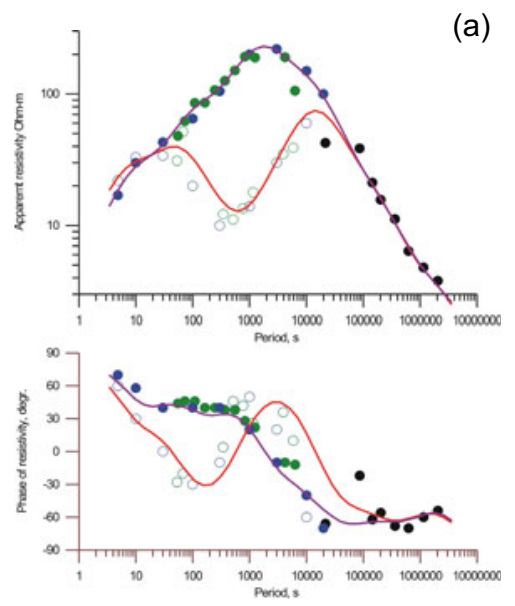

(b)

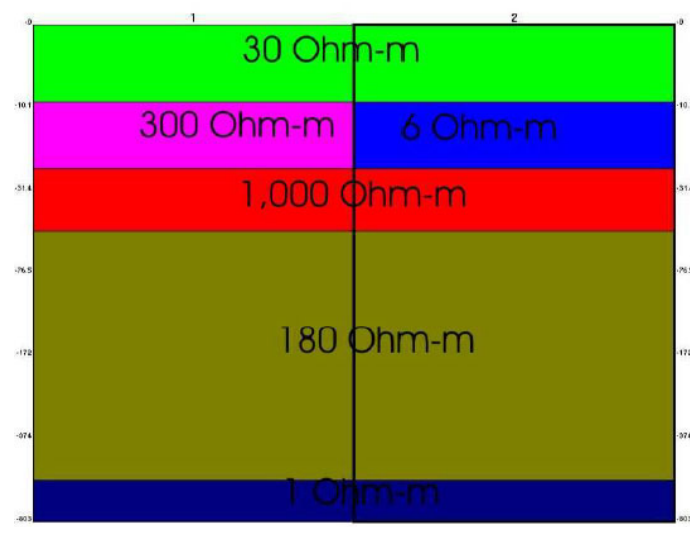

Fig. 7. The combined MT and MV apparent resistivities at two preferential directions across (black) and alongside (red) in the center of EMTESZ profiles (a) and their both 1D inversion models (b) (after Semenov et al. 2005).

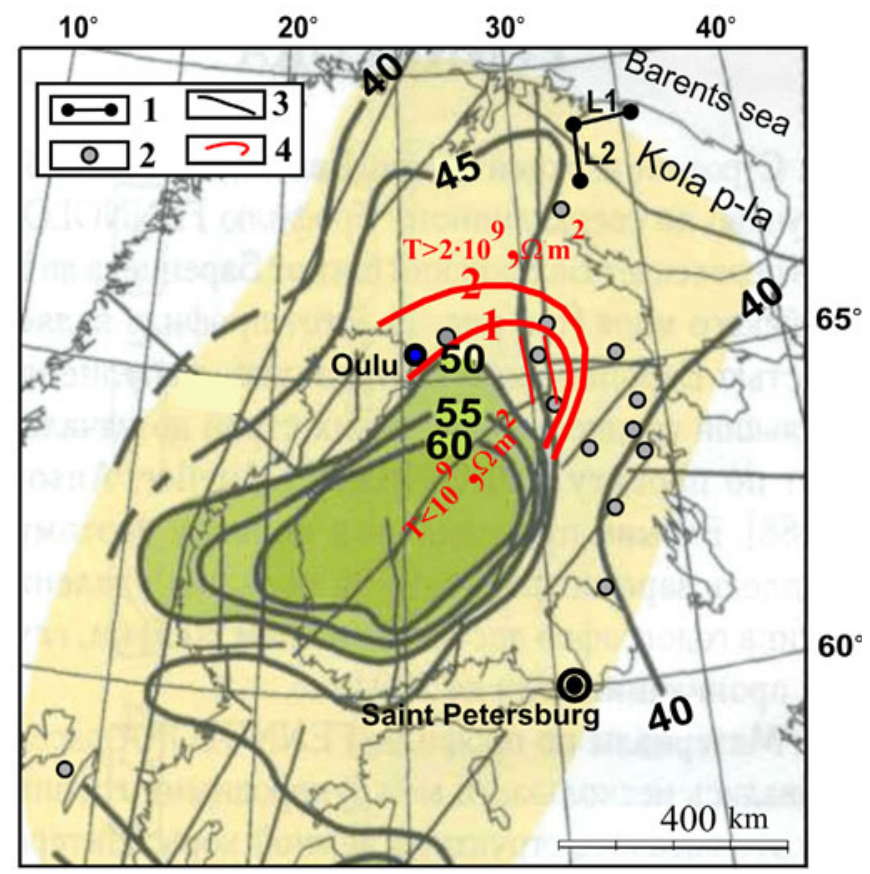

Fig. 8. The gradient of transversal resistance $T\left[\Omega \cdot \mathrm{m}^{2}\right]$ of the crust at the depth interval $10-60 \mathrm{~km}$ (red lines) coinciding with boundary of the deepest Moho zone in Europe (black lines) (after Zhamaletdinov 2011, Zhamaletdinov et al. 2011). 
The fifth international project was the LS-MVS (2009-2012). Five countries took part in these investigations. New MVS method was tested successfully in Central Europe using the data of geomagnetic observatories (Semenov et al. 2011). Thus, distributions of the induction arrows as well as response functions were estimated in Poland for the period range from 3 hours to one day. This new information is analyzed now.

The original method to analyze the spatial distribution of induction arrows has been proposed recently by Jóźwiak (2012). Known arrows were recalculated to the scalar tippers and then into a ratio of the horizontal field at different points relative to a point at infinity. The Hilbert's transformation was used for this aim. These red zones are characterized by high conductance (Fig. 9). The obtained results are in good agreement with the geological knowledge.

Recently, sounding results using data of the global geomagnetic observatory net were published by Praus et al. (2011). A review of electromagnetic study of lithospheric structure around the TESZ was made by Jóźwiak (2013).

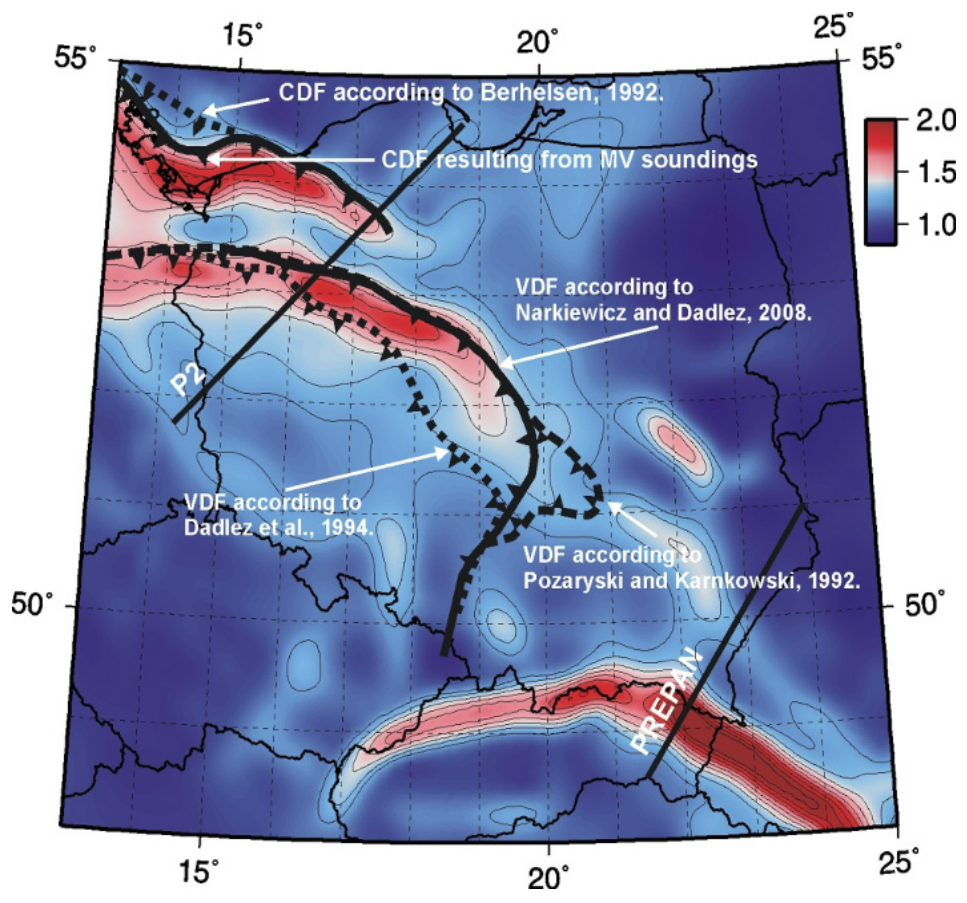

Fig. 9. Scheme of the spatial distribution of the conductive structures (red) basing on the hypothetical locations of the Caledonian and Variscan deformation fronts (right, scale in log of Siemens) (after Jóźwiak 2012). 


\section{CONCLUSIONS AND DISCUSSION}

The development of generalized magnetovariation sounding theory significantly expanded possibilities of induction soundings in the period range from hours to a couple of days. The regional investigations by this method allow estimating additionally gradients of response functions including information about electrical inhomogeneities in the upper mantle (up to about $500 \mathrm{~km}$ ) that was not made earlier. The induction sounding results are not stable: their long period variations can be caused by internal sources while variations with shorter periods by an abrupt change of the external field source. It is a reason why the results of formal inversions may be changeable in time. Besides, the forward spherical modeling has shown that sounding results using different sources and sounding methods above deep inhomogeneities can be essentially different. However, the sounding results of GMV and MT methods remain close even over significant irregularities in the Earth.

The methodic peculiarities of the mantle soundings are connected with combinations of two induction methods with tensor (MT) and scalar (GMV) or vectoral (GDS) apparent resistivities. The choice of destinations in the MT soundings to compare with the MV ones is promising if the resistivity of medium assumed to be a tensor rather than the impedance. It was shown that the conductance is most reliable for comparison between sounding results obtained by different investigators. An attractive but expensive method is that of controlled sources (like MGD generator) used in Russia for the deep soundings practically on a non-conductive surface. A lot of problems connected with natural sources disappear in such soundings, but requirements concerning the theory of the methods are not reduced.

Four of the five international projects in Central and Northern Europe performed areal studies. The results of the BEAR experiment increased the knowledge about the electrical structure of the Earth's crust at the Fennoscandian Shield (Korja et al. 2002). But another goal of the experiment - the search for a possible existence of the asthenosphere in the upper mantle - did not get a clear answer. Moreover, different research groups have come to some conflicting conclusions from the same experimental data. According to the St. Petersburg group an intermediate conductive layer associated with partial melting of rocks is clearly recorded in the depth range of $200-400 \mathrm{~km}$ and its average value of the longitudinal conductance reaches $8 \mathrm{kS}$ (Vardanyants and Kovtun 2009). This conclusion is not contrary to the interpretation of the seismic data which has a slightly different depth about 100-150 km (Abramovitz et al. 2002). On the other hand, the interpretations made by other creative teams on the basis of the same experimental data assume absence of any asthenosphere under the Fennoscandian shield 
or it is extremely weak on the background of the confidence limits (Varentsov et al. 2002, Sokolova and Varentsov 2007). The last interpretation of the BEAR data coincides with interpretation made by Vanyan et al. (2002). Discrepancies between the interpretations may be explained by sharp electrical inhomogeneity of the crust at that resistive region and high latitudes with complicated source field. It would be interesting to take into account the deep borehole in Karelia, where extremely solid rocks were met at the depth about $12 \mathrm{~km}$.

The two main results of the CEMES project have fixed gradients of the total conductance in the mantle at 300 and $770 \mathrm{~km}$ depths, coinciding with the TESZ. Now physical explanation of this effect is absent. It could be attributed to the methodological inaccuracies or errors of observations, but a similar inverse pattern was observed on the results of seismic tomography (Piromallo and Morelli 2003). Reverses of the overabundant mass densities were also established at about $700 \mathrm{~km}$ depth by a rigorous analysis of the satellite data (Martinec and Pěč 1990). These phenomena require additional investigations as well as influence of the Earth magnetic permeability on the MV soundings and analysis of induction arrows at the period range of 3-30 hours.

The FENICS project has allowed estimating the position of the transversal resistance gradients changing twice at the boundary of the deepest (about $50-60 \mathrm{~km}$ ) area of the Moho in Northern Europe. These northern boundaries (gradient zones) of electrical and gravity anomalies coincide. Besides, the $1 \mathrm{D}$ inversions show the lower crust resistivity (about $10^{5} \Omega \cdot \mathrm{m}$ ), which is at least two orders more than for other shields in Europe and Canada. This high resistivity is coinciding well with the laboratory investigations for depths of $10-50 \mathrm{~km}$ (Zhamaletdinov et al. 2011) but is not consistent with previous studies (Vanyan et al. 2001) and other inversion results (Sokolova and Varentsov 2007).

Acknowledgments. The research was partially supported within statutory activities No. 3841/E-41/S/2014 of the Ministry of Science and Higher Education of Poland and by National Science Centre (NCN), Poland, grant No. 2011/01/B/ST1O/07046. Author is thankful to prof. A.A. Zhamaletdinov for discussions. 


\section{References}

Aboul-Atta, O.A., and W.M. Boerner (1975), Vectoral impedance identity for the natural dependence of harmonic fields on closed boundaries, Canadian J. Phys. 53, 15, 1404-1407, DOI: 10.1139/p75-179.

Abramovitz, T., H. Thybo, and E. Perchuć (2002), Tomographic inversion of seismic P- and S-wave velocities from the Baltic Shield based on FENNOLORA data, Tectonophysics 358, 1-4, 151-174, DOI: 10.1016/S0040-1951 (02)00422-5.

Banks, R.J. (1969), Geomagnetic variations and the electrical conductivity of the upper mantle, Geophys. J. Roy. Astr. Soc. 17, 5, 457-487, DOI: 10.1111/ j.1365-246X.1969.tb00252.x.

Bates, R.H.T., W.M. Boerner, and G.R. Dunlop (1976), An extended Rytov approximation and its significance for remote sensing and inverse scattering, Opt. Commun. 18, 4, 421-423, DOI: 10.1016/0030-4018(76)90285-6.

Becken, M., and L.B. Pedersen (2003), Transformation of VLF anomaly maps into apparent resistivity and phase, Geophysics 68, 2, 497-505, DOI: 10.1190/ 1.1567217.

Becken, M., O. Ritter, and H. Burkhardt (2008), Mode separation of magnetotelluric responses in three-dimensional environments, Geophys. J. Int. 172, 1, 6786, DOI: 10.1111/j.1365-246X.2007.03612.x.

Berdichevsky, M.N., and M.S. Zhdanov (1981), Interpretation of Anomalies Alternating Electromagnetic Field of the Earth, Nedra, Moscow (in Russian).

Berdichevsky, M.N., L.L. Vanyan, and E.B. Fainberg (1969), The frequency sounding of the Earth using spherical analysis results of geomagnetic variations, Geomagn. Aeron. 9, 372-374 (in Russian).

Cagniard, L. (1953), Basic theory of the magneto-telluric method of geophysical prospecting, Geophysics 18, 3, 605-635, DOI: 10.1190/1.1437915.

Dmitriev, V.I., and M.N. Berdichevsky (2002), A generalized model of impedance, Izv. Phys. Solid Earth 38, 10, 897-903.

Egbert, G.D., and J.R. Booker (1992), Very long period magnetotellurics at Tucson observatory: Implications for mantle conductivity, J. Geophys. Res. 97, B11, 15099-15112, DOI: 10.1029/92JB01251.

Ernst, T., H. Brasse, V. Cherv, N. Hoffmann, J. Jankowski, W. Jóźwiak, A. Kreutzmann, A. Neska, N. Palshin, L.B. Pedersen, M. Smirnov, E. Sokolova, and I.M. Varentsov (2008), Electromagnetic images of the deep structure of the Trans-European Suture Zone beneath Polish Pomerania, Geophys. Res. Lett. 35, 15, L15307, DOI: 10.1029/2008GL034610.

Fujii, I., and A. Schultz (2002), The 3D electromagnetic response of the Earth to ring current and auroral oval excitation, Geophys. J. Int. 151, 3, 689-709, DOI: 10.1046/j.1365-246X.2002.01775.x. 
Grad, M., T. Tiira, and ESC Working Group (2007), The Moho depth of the European plate, European Seismological Commission, Warsaw-Helsinki, http:// www.seismo.helsinki.fi/mohomap/; http://www.igf.fuw.edu.pl/mohomap/.

Guglielmi, A.V., and M.B. Gokhberg (1987), On the magnetotelluric sounding in the seismically active areas, Izv. Phys. Solid Earth 33, 11, 122-123 (in Russian).

Guterch, A., M. Grad, T. Janik, R. Materzok, U. Luosto, J. Yliniemi, E. Lück, A. Schulze, and K. Förste (1994), Crustal structure of the transition zone between Precambrian and Variscan Europe from new seismic data along LT-7 profile (NW Poland and eastern Germany), C. R. Acad. Sci. II Paris 319, 12, 1489-1496.

Harrington, R.F. (1961), Time-Harmonic Electromagnetic Fields, McGraw-Hill, New York, 480 pp.

Ingerov, A.I., I.I. Rokityansky, and V.I. Tregubenko (1999), Forty years of MTS studies in the Ukraine, Earth Planets Space 51, 10, 1127-1133.

Janik, T., J. Yliniemi, M. Grad, H. Thybo, T. Tiira, and POLONAISE P2 Working Group 1 (2002), Crustal structure across the TESZ along POLONAISE'97 seismic profile P2 in NW Poland, Tectonophysics 360, 1-4, 129-152, DOI: 10.1016/S0040-1951(02)00353-0.

Jóźwiak, W. (2012), Large-scale crustal conductivity pattern in Central Europe and its correlation to deep tectonic structures, Pure Appl. Geophys. 169, 10, 1737-1747, DOI: 10.1007/s00024-011-0435-7.

Jóźwiak, W. (2013), Electromagnetic study of lithospheric structure in the marginal zone of East European Craton in NW Poland, Acta Geophys. 61, 5, 11011129, DOI: 10.2478/s11600-013-0127-z.

Kiss, J., L. Szarka, and E. Prácser (2005), Second-order magnetic phase transition in the Earth, Geophys. Res. Lett. 32, 24, L24310, DOI: 10.1029/2005GL 024199.

Korja, T. (2007), How is the European lithosphere imaged by magnetotellurics? Surv. Geophys. 28, 2-3, 239-272, DOI: 10.1007/s10712-007-9024-9.

Korja, T., M. Engels, A.A. Zhamaletdinov, A.A. Kovtun, N.A. Palshin, M.Yu. Smirnov, A.D. Tokarev, V.E. Asming, L.L. Vanyan, I.L. Vardaniants, and the BEAR Working Group (2002), Crustal conductivity in Fennoscandia a compilation of a database on crustal conductance in the Fennoscandian Shield, Earth Planets Space 54, 5, 535-558.

Kuckes, A.F. (1973), Relations between electrical conductivity of a mantle and fluctuating magnetic fields, Geophys. J. Roy. Astr. Soc. 32, 1, 119-130, DOI: 10.1111/j.1365-246X.1973.tb06523.x.

Kuckes, A.F., A.G. Nekut, and B.G. Thompson (1985), A geomagnetic scattering theory for evaluation of the Earth structure, Geophys. J. Roy. Astr. Soc. 83, 2, 319-330, DOI: 10.1111/j.1365-246X.1985.tb06489.x. 
Kuvshinov, A.V. (2012), Deep electromagnetic studies from land, sea, and space: Progress status in the past 10 years, Surv Geophys. 33, 1, 169-209, DOI: 10.1007/s10712-011-9118-2.

Kuvshinov, A., H. Utada, D. Avdeev, and T. Koyama (2005), 3-D modelling and analysis of Dst C-responses in the North Pacific Ocean region, revisited, Geophys. J. Int. 160, 2, 505-526, DOI: 10.1111/j.1365-246X.2005.02477.x.

Leontovich, M.A. (1948), On approximate boundary conditions for an electromagnetic field on the surface of highly conductive bodies. In: Issledovania po Rasprostraneniu Radiovoln, Acad. Sci. USSR, Moscow, 5-12 (in Russian).

Logvinov, I.M. (2002), Applying the horizontal spatial gradient method for the deep conductivity estimations in the Ukraine, Acta Geophys. Pol. 50, 4, 567-573.

Martinec, Z., and K. Pěč (1990), The influence of the core-mantle boundary irregularities on the mass density distribution inside the Earth. In: A. Vogel, C.O. Ofoegbu, R. Gorenflo, and B. Ursin (eds.), Geophysical Data Inversion. Methods and Applications, Proc. 7th Int. Math. Geophys. Seminar, 8-11 February 1989, Free University of Berlin, 233-256, DOI: 10.1007/ 978-3-322-89416-8_15.

Nowożyński, K. (2004), Estimation of magnetotelluric transfer functions in the time domain over a wide frequency band, Geophys. J. Int. 158, 1, 32-41, DOI: 10.1111/j.1365-246X.2004.02288.x.

Olsen, N. (1992), Day-to-day C-response estimation for Sq from $1 \mathrm{cpd}$ to $6 \mathrm{cpd}$ using the Z:Y-method, J. Geomag. Geoelectr. 44, 6, 433-447, DOI: 10.5636/ jgg.44.433.

Olsen, N. (1998), The electrical conductivity of the mantle beneath Europe derived from C-responses from 3 to $720 \mathrm{hr}$, Geophys. J. Int. 133, 2, 298-308, DOI: 10.1046/j.1365-246X.1998.00503.x.

Olsen, N. (1999a), Long-period (30 days - 1 year) electromagnetic sounding and the electrical conductivity of the lower mantle beneath Europe, Geophys. J. Int. 138, 1, 179-187, DOI: 10.1046/j.1365-246x.1999.00854.x.

Olsen, N. (1999b), Induction studies with satellite data, Surv. Geophys. 20, 3-4, 309340, DOI: $10.1023 / \mathrm{A}: 1006611303582$.

Oraevsky, V.N., N.M. Rotanova, T.N. Bondar, D.Yu. Abramova, and V.Yu. Semenov (1993), On the radial geoelectrical structure of the mid-mantle from magnetovariational sounding using MAGSAT data, J. Geomagn. Geoelectr. 45, 11-12, 1415-1423.

Pek, J. (2002), Spectral magnetotelluric impedances for an anisotropic layered conductor, Acta Geophys. Pol. 50, 4, 619-643.

Pek, J., and F.A.M. Santos (2002), Magnetotelluric impedances and parametric sensitivities for 1-D anisotropic layered media, Comput. Geosci. 28, 8, 939950, DOI: 10.1016/S0098-3004(02)00014-6. 
Petrishchev, M.S., and V.Yu. Semenov (2013), Secular variations of the Earth's apparent resistivity, Earth Planet Sci. Lett. 361, 1-6, DOI: 10.1016/j.epsl. 2012.11.027.

Piromallo, C., and A. Morelli (2003), P wave tomography of the mantle under the Alpine-Mediterranean area, J. Geophys. Res. 108, B2, 2065, DOI: 10.1029/ 2002JB001757.

Praus, O., J. Pěčova, V. Červ, S. Kováčiková, J. Pek, and J. Velímský (2011), Electrical conductivity at mid-mantle depths estimated from the data of Sq and long period geomagnetic variations, Stud. Geophys. Geod. 55, 2, 241-264, DOI: $10.1007 / \mathrm{s} 11200-011-0014-5$.

Roberts, R.G. (1984), The long-period electromagnetic response of the Earth, Geophys. J. Roy. Astr. Soc. 78, 2, 547-572, DOI: 10.1111/j.1365-246X. 1984.tb01963.x.

Roberts, R.G. (1986), The deep electrical structure of the Earth, Geophys. J. Int. 85, 3, 583-600, DOI: 10.1111/j.1365-246X.1986.tb04534.x.

Rokitjansky, I.I. (1982), Geomagnetic Investigation of the Earth's Crust and Mantle, Springer, Berlin, $381 \mathrm{pp}$.

Rytov, S.M. (1940), Skin-effect calculations by the disturbance method, J. Exp. Theor. Phys. 10, 2, 180-189 (in Russian).

Schmucker, U. (1970), Anomalies of Geomagnetic Variations in the Southwestern United States, University of California Press, Berkeley, 165 pp.

Schmucker, U. (1999a), A spherical harmonic analysis of solar daily variations in the years 1964-1965: response estimates and source fields for global induction - I. Methods, Geophys. J. Int. 136, 2, 439-454, DOI: 10.1046/j.1365246X.1999.00742.x.

Schmucker, U. (1999b), A spherical harmonic analysis of solar daily variations in the years 1964-1965: response estimates and source fields for global induction - II. Results, Geophys. J. Int. 136, 2, 455-476, DOI: 10.1046/j.1365246X.1999.00743.x.

Schmucker, U. (2003), Horizontal spatial gradient sounding and geomagnetic depth sounding in the period range of daily variations. In: A. Hördt and J.B. Stoll (eds.), Protokoll über das 20. Kolloquium elektromagnetische Tiefenforschung, 29.09-3.10.2003, Königstein, Deutschland, 228-237.

Schultz, A. (1990), On the vertical gradient and associated heterogeneity in mantle electrical conductivity, Phys. Earth Planet. In. 64, 1, 68-86, DOI: 10.1016/ 0031-9201(90)90006-J.

Schultz, A., and J.C. Larsen (1987), On the electrical conductivity of the mid-mantle - I. Calculation of equivalent scalar magnetotelluric response functions, Geophys. J. Int. 88, 3, 733-761, DOI: 10.1111/j.1365-246X.1987. tb01654.x.

Schultz, A., and J.C. Larsen (1990), On the electrical conductivity of the mid-mantle - II. Delineation of heterogeneity by application of extremal inverse solu- 
tions, Geophys. J. Int. 101, 3, 565-580, DOI: 10.1111/j.1365-246X.1990. tb05571.x.

Schultz, A., and T.S. Zhang (1994), Regularized spherical harmonic analysis and the 3-D electromagnetic response of the Earth, Geophys. J. Int. 116, 1, 141156, DOI: 10.1111/j.1365-246X.1994.tb02133.x.

Schultz, A., R.D. Kurtz, A.D. Chave, and A.G. Jones (1993), Conductivity discontinuities in the upper mantle beneath a stable craton, Geophys. Res. Lett. 20, 24, 2941-2944, DOI: 10.1029/93GL02833.

Semenov, V.Yu. (1988), Analysis of magnetotelluric data during the anisotropic media sounding, Russ. Geol. Geophys. 10, 121-125 (in Russian).

Semenov, V.Yu. (1989), Evaluation of mantle conductivity beneath northern hemisphere continents, Izv. - Phys. Solid Earth 25, 3, 221-226 (in Russian).

Semenov, V.Yu. (2000), On the apparent resistivity in magnetotelluric sounding, Izv. - Phys. Solid Earth 36, 1, 99-100.

Semenov, V.Yu., and W. Jóźwiak (1999), Model of the geoelectrical structure of the mid- and lower mantle in the Europe-Asia region, Geophys. J. Int. 138, 2, 549-552, DOI: 10.1046/j.1365-246X.1999.00888.x.

Semenov, V.Yu., and W. Jóźwiak (2005), Estimation of the upper mantle electric conductance at the Polish margin of the East European platform, Izv. Phys. Solid Earth 41, 4, 326-332.

Semenov, V.Yu., and W. Jóźwiak (2006), Lateral variations of the mid-mantle conductance beneath Europe, Tectonophysics 416, 1-4, 279-288, DOI: 10.1016/j.tecto.2005.11.017.

Semenov, V.Yu., and M. Rodkin (1996), Conductivity structure of the upper mantle in an activ subduction zone, J. Geodynamics 21, 4, 355-364, DOI: 10.1016/0264-3707(95)00038-0.

Semenov, V.Yu., and V.N. Shuman (2010), Impedances for induction soundings of the Earth's mantle, Acta Geophys. 58, 4, 527-542, DOI: 10.2478/s11600010-0003-z.

Semenov, V.Yu., T. Ernst, K. Nowożyński, J. Pek, and EMTESZ WG (2005), Estimation of the deep geoelectrical structure beneath TESZ in NW Poland, Publs. Inst. Geophys. Pol. Acad. Sci. C-95, 386, 63-65.

Semenov, V.Yu., J. Pek, A. Ádám, W. Jóźwiak, B. Ladanyvskyy, I.M. Logvinov, P. Pushkarev, J. Vozar, and Experimental Team of CEMES project (2008), Electrical structure of the upper mantle beneath Central Europe: Results of the CEMES project, Acta Geophys. 56, 4, 957-981, DOI: 10.2478/s11600008-0058-2.

Semenov, V.Yu., B. Ladanivskyy, and K. Nowożyński (2011), New induction sounding tested in Central Europe, Acta Geophys. 59, 5, 815-832, DOI: 10.2478/s11600-011-0030-4. 
Semenov, V.Yu., M. Hvoždara, and J. Vozar (2013), Modeling of deep magnetovariation soundings on the rotating Earth, Acta Geophys. 61, 2, 264-280, DOI: $10.2478 / \mathrm{s} 11600-012-0086-9$.

Senior, T.B.A., and J.L. Volakis (1995), Approximate Boundary Conditions in Electromagnetics, IEE Press, London, $353 \mathrm{pp}$.

Shuman, V.N. (1999), Scalar local impedance conditions and the impedance tensor in processing and interpretation of a magnetotelluric experiment, Geophys. J. 19, 361-385.

Shuman, V. (2003), The general theory of geoelectromagnetic sounding systems accounting the electrodynamics of spherical sources. In: 3DEM III Workshop, February 2003, Adelaide, USA, ASEG Ext. Abstr., 1-7, DOI: 10.1071/ ASEG2003_3DEMab015.

Shuman, V.N. (2007), Imaginary surface vectors in multidimensional inverse problems of geoelectrics, Izv. - Phys. Solid Earth 43, 3, 205-210, DOI: $10.1134 / \mathrm{S} 1069351307030044$.

Shuman, V., and S. Kulik (2002), The fundamental relations of impedance type in general theories of the electromagnetic induction studies, Acta Geophys. Pol. 50, 4, 607-618.

Sokolova, E.Yu., I.M. Varentsov, and BEAR Working Group (2007), Deep array electromagnetic sounding on the Baltic Shield: External excitation model and implications for upper mantle conductivity studies, Tectonophysics 445, 1-2, 3-25, DOI: 10.1016/j.tecto.2007.07.006.

Szarka, L., A. Franke, E. Prácser, and J. Kiss (2007), Hypothetical mid-crustal models of second-order magnetic phase transition. In: 4th Int. Symp. on ThreeDimensional Electromagnetics, 27-30 September 2007, Freiberg, Germany.

Tikhonov, A.N. (1950), On determining electrical characteristics of the deep layers of the Earth's crust, Dokl. AN USSR 73, 2, 295-297 (in Russian).

Transactions... (1973), Transactions of the 2nd IAGA Scientific Assembly, Kyoto, Japan, 1973, IAGA Bull. 35, 189 pp.

Vanyan, L., B. Tezkan, and N. Palshin (2001), Low electrical resistivity and seismic velocity at the base of the upper crust as indicator of rheologically weak layer, Surv. Geophys. 22, 2, 131-154, DOI: 10.1023/A:1012937410685.

Vanyan, L.L., V.A. Kuznetsov, T.V. Lyubetskaya, N.A. Palshin, T. Korja, I. Lahti, and the BEAR Working Group (2002), Electrical conductivity of the crust beneath Central Lapland, Izv. - Phys. Solid Earth 38, 10, 798-815.

Vardanyants, I.L., and A.A. Kovtun (2009), The study of the possible existence of asthenosphere on the territory of Fennoscandian shield by the BEAR data. In: Complex Geological-Geophysical Models of Ancient Shields, Geological Inst. of the Kola Science Centre, Russ. Acad. Sc., Apatity, 15-18.

Varentsov, I.M., M. Engels, T. Korja, M.Yu. Smirnov, and the BEAR Working Group (2002), The generalized geoelectric model of Fennoscandia: A challenging database for long-period 3D modeling studies within the Baltic 
electromagnetic array research (BEAR) Project, Izv. - Phys. Solid Earth 38, 11, 855-896 (in Russian).

Vozar, J., and V.Yu. Semenov (2010), Compatibility of induction methods for mantle soundings, J. Geophys. Res. 115, B3, B03101, DOI: 10.1029/2009JB 006390.

Wait, J.R. (1954), On the relation between telluric currents and the Earth's magnetic field, Geophysics 19, 2, 281-289, DOI: 10.1190/1.1437994.

Weckmann, U., O. Ritter, and V. Haak (2003), Images of the magnetotelluric apparent resistivity tensor, Geophys. J. Int. 155, 2, 456-468, DOI: 10.1046/ j.1365-246X.2003.02062.x.

Woods, D.V., and F.E.M. Lilley (1979), Geomagnetic induction in Central Australia, J. Geomag. Geoelectr. 31, 4, 449-458, DOI: 10.5636/jgg.31.449.

Zhamaletdinov, A.A. (1996), Graphite in the Earth's crust and electrical conductivity anomalies, Izv. - Phys. Solid Earth 32, 4, 272-288.

Zhamaletdinov, A.A. (2005), Khibiny MHD experiment: The 30th anniversary, Izv. - Phys. Solid Earth 41, 9, 737-742.

Zhamaletdinov, A.A. (2011), The new data on the structure of the continental crust based on the results of electromagnetic sounding with the use of powerful controlled sources, Dokl. Earth Sci. 438, 2, 798-802, DOI: 10.1134/ S1028334X11060146.

Zhamaletdinov, A.A., A.N. Shevtsov, T.G. Korotkova, Yu.A. Kopytenko, V.S. Ismagilov, M.S. Petrishev, B.V. Efimov, M.B. Barannik, V.V. Kolobov, P.I. Prokopchuk, M.Yu. Smirnov, S.A. Vagin, M.I. Pertel, E.D. Tereshchenko, A.N. Vasil'ev, V.F. Grigoryev, M.B. Gokhberg, V.I. Trofimchik, Yu.M. Yampolsky, A.V. Koloskov, A.V. Fedorov, and T. Korja (2011), Deep electromagnetic sounding of the lithosphere in the Eastern Baltic (Fennoscandian) shield with high-power controlled sources and industrial power transmission lines (FENICS experiment), Izv. - Phys. Solid Earth 47, 1, 2-22, DOI: 10.1134/S1069351311010149.

Received 5 December 2013 Received in revised form 20 January 2014 Accepted 22 January 2014 